\title{
Ultrasound Image Denoising Using Generative Adversarial Networks with residual dense connectivity and weighted joint loss
}

\author{
Lun Zhang ${ }^{1,2}$, Junhua Zhang ${ }^{\text {Corresp. } 1}$ \\ ${ }^{1}$ School of Information Science and Engineering, Yunnan University, Kunming, Yunnan, China \\ 2 Yunnan Vocational Institute of Energy Technology, Qujing, Yunnan, China \\ Corresponding Author: Junhua Zhang \\ Email address: jhzhang@ynu.edu.cn
}

Background.

Ultrasound imaging has been recognized as a powerful tool in clinical diagnosis. Nonetheless, the presence of speckle noise degrades the signal-to-noise of ultrasound images. Various denoising algorithms cannot fully reduce speckle noise and retain image features well for ultrasound image. With the development of deep learning, the application of deep learning in ultrasound image denoising has attracted more and more attention in recent years.

Methods.

In the article, we propose a generative adversarial network with residual dense connectivity and weighted joint loss (GAN-RW) to avoid the limitations of traditional image denoising algorithms and surpass the most advanced performance of ultrasound image denoising. The denoising network is based on U-Net architecture which includes four encoder and four decoder modules. Each of the encoder and decoder is replaced with residual dense connectivity and $B N$ to remove speckle noise. The discriminator network applies a series of convolutional layers to identify differences between the translated images and the desired modality. In the training processes, we introduce a joint loss function consisting of a weighted sum of the L1 loss function, binary cross-entropy with a logit loss function and perceptual loss function.

Results.

We split experiments into two parts. First, experiments were performed on Berkeley segmentation (BSD68) datasets corrupted by simulated speckle. Compared with the eight existing denoising algorithms, the GAN-RW achieved the most advanced despeckling performance in terms of the peak signal-to-noise ratio (PSNR), structural similarity (SSIM) and subjective visual effect. When the noise level was 15 , the average value of the GAN-RW increased by approximately $3.58 \%$ and $1.23 \%$ for PSNR and SSIM, respectively. When the noise level was 25 , the average value of the GAN-RW increased by approximately $3.08 \%$ and $1.84 \%$ for PSNR and SSIM, respectively. When the noise level was 50 , the average value of the GAN-RW increased by approximately $1.32 \%$ and $1.98 \%$ for PSNR and SSIM, respectively. Second, experiments were performed on the ultrasound images of lymph nodes, the foetal head and the brachial plexus. The proposed method shows higher subjective visual effect when verifying on the ultrasound images. In the end, through statistical analysis, the GAN-RW achieved the highest mean rank in the Friedman test. 


\section{Ultrasound Image Denoising Using Generative}

\section{Adversarial Networks with residual dense connectivity}

\section{3 and weighted joint loss}

4

5 Lun Zhang ${ }^{1,2}$, Junhua Zhang ${ }^{1}$

6

$7 \quad{ }^{1}$ School of Information Science and Engineering, Yunnan University, Kunming, Yunnan, China

$8{ }^{2}$ Yunnan Vocational Institute of Energy Technology, Qujing, Yunnan, China

10 Corresponding Author:

11 Junhua Zhang ${ }^{1}$

12 Dongwaihuan South Rd Chenggong District, Kunming, Yunnan, 650504, China

13 Email address: jhzhang@ynu.edu.cn 


\section{Abstract}

\section{Background.}

26 Ultrasound imaging has been recognized as a powerful tool in clinical diagnosis. Nonetheless,

27 the presence of speckle noise degrades the signal-to-noise of ultrasound images. Various

28 denoising algorithms cannot fully reduce speckle noise and retain image features well for

29 ultrasound image. With the development of deep learning, the application of deep learning in

30 ultrasound image denoising has attracted more and more attention in recent years.

\section{Methods.}

32 In the article, we propose a generative adversarial network with residual dense connectivity and weighted joint loss (GAN-RW) to avoid the limitations of traditional image denoising algorithms

34 and surpass the most advanced performance of ultrasound image denoising. The denoising

35 network is based on U-Net architecture which includes four encoder and four decoder modules.

36 Each of the encoder and decoder is replaced with residual dense connectivity and BN to remove

37 speckle noise. The discriminator network applies a series of convolutional layers to identify

38 differences between the translated images and the desired modality. In the training processes, we

39 introduce a joint loss function consisting of a weighted sum of the L1 loss function, binary cross-

40 entropy with a logit loss function and perceptual loss function.

\section{Results.}

42 We split experiments into two parts. First, experiments were performed on Berkeley

43 segmentation (BSD68) datasets corrupted by simulated speckle. Compared with the eight

44 existing denoising algorithms, the GAN-RW achieved the most advanced despeckling

45 performance in terms of the peak signal-to-noise ratio (PSNR), structural similarity (SSIM) and

46 subjective visual effect. When the noise level was 15 , the average value of the GAN-RW 
47 increased by approximately $3.58 \%$ and $1.23 \%$ for PSNR and SSIM, respectively. When the noise

48 level was 25 , the average value of the GAN-RW increased by approximately $3.08 \%$ and $1.84 \%$

49 for PSNR and SSIM, respectively. When the noise level was 50, the average value of the GAN-

50 RW increased by approximately $1.32 \%$ and $1.98 \%$ for PSNR and SSIM, respectively. Second,

51 experiments were performed on the ultrasound images of lymph nodes, the foetal head and the

52 brachial plexus. The proposed method shows higher subjective visual effect when verifying on

53 the ultrasound images. In the end, through statistical analysis, the GAN-RW achieved the highest

54 mean rank in the Friedman test.

55

56 Keywords deep learning, ultrasound image denoising, generative adversarial network

57

58

59

60

61

62

63

64

65

66

67

68

69 


\section{Introduction}

72 Ultrasound has been widely used in clinical diagnosis. Compared with CT and MRI, it has the

73 advantages of cost-effectiveness and non-ionizing radiation. However, due to the coherent

74 nature, speckle noise is inherent in ultrasound images (Singh et al., 2017). The speckle noise is

75 the primary cause of low contrast resolution and the signal-to-noise ratio. It makes image

76 processing and analysis more challenging, such as image classification and segmentation.

77 Therefore, eliminating speckle noise is of great significance for improving the ultrasound images

78 signal-to-noise ratio and diagnosing disease accurately.

79 There are various traditional methods for image denoising, which include frequency

80 domain, time-domain and joint time-domain/frequency-domain methods. Among the traditional

81 methods, the most widely used denoising method is based on wavelets (Jaiswal et al., 2014;

82 Srivastava et al., 2016; Gupta et al., 2004). Shih et al. proposed an iterated two-band filtering

83 method to solve the selective image smoothing problem (Shih et al., 2003). Yue et al. introduced

84 a novel nonlinear multiscale wavelet diffusion for speckle noise removal and edge enhancement,

85 which proved that this method is better than wavelet-transform alone in removing speckle noise

86 (Yue et al., 2006). Among the above methods, speckle noise is transformed into additive noise

87 and removed. Because speckle noise is not purely multiplicative noise, the selection of wavelets

88 is based on experience, which creates artefacts. Traditional methods based on the spatial domain

89 include the Kuan filter (Kuan et al.,1987), speckle reducing anisotropic diffusion filter (Yu et al.,

90 2002) and Frost filter (Frost et al., 1982). These methods mainly use local pixel comparison. The

91 nonlocal means (NLM) method was proposed which is based on a nonlocal averaging of all

92 pixels in the image (Buades et al., 2005). However, the NLM filter cannot preserve the fine 
93 details and edge features in the image. Dabov et al. proposed a block-matching and 3D

94 transform-domain collaborative filtering (BM3D) method, which reduced the computing time

95 and effectively suppressed noise by grouping 3D data arrays of similar 2D image fragments

96 (Dabov et al., 2007). However, the disadvantage of these methods is that they cannot maintain a

97 balance between noise suppression and image detail preservation.

98 The development of deep learning provides a perfectly feasible solution for image denoising.

99 Zhang et al. (Zhang et al., 2017) introduced feed-forward denoising convolutional neural

100 network (DnCNN), where residual learning (He et al., 2016) was adopted to separate noise from

101 noisy image and batch normalization (BN) (Salimans et al., 2016) was integrated to speed up the

102 training process and boost the denoising performance. Using the small medical image datasets,

103 Jifara et al. designed a denoising convolutional neural network with residual learning and $\mathrm{BN}$ for

104 medical image denoising (DnCNN-Enhanced) (Jifara et al., 2019). More specifically, they used

105 residual learning by multiplying a very small constant and added it to better approximate the

106 residual to improve performance. Tian et al. proposed a novel algorithm called a batch-

107 renormalization denoising network (BRDNet) for image denoising (Tian et al., 2020). This

108 network combines two networks to expand the width to capture more feature information.

109 Meanwhile, BRDNet adopted BN to address small mini-batch problems and dilated convolution

110 to enlarge the receptive field to extract more feature information. In addition to feed-forward

111 denoising algorithm, there are some algorithms based on the encoder-decoder network. The U-

112 Net is the most widely used encoder-decoder network which used the segmentation of

113 biomedical images (Ronneberger et al., 2015). These are various algorithms based on U-Net for

114 image processing, such as U-Net++ (Zhou et al., 2019), residual-dilated-attention-gate network

115 (RDAU-Net) (Zhuang et al., 2019), Wasserstein GAN algorithm (RDA-UNET-GAN) (Negi et 
116 al., 2020), Attention Gate-Dense Network-Improved Dilation Convolution-U-Net (ADID-

117 UNET) (Raj et al., 2021), VGG-UNet (Fawakherji et al., 2019), Ens4B-UNet (Abedalla et al.,

118 2021) and so on. Park et al. designed a densely connected hierarchical image denoising network

119 (DHDN) for removing additive white Gaussian noise of natural images (Park et al., 2019).

120 Based on the U-Net, it applied the hierarchical architecture of the encoder-decoder module with

121 dense connectivity and residual learning to solve the vanishing-gradient problem. Guo et al.

122 suggested training a convolutional blind denoising network (CBDNet) using noisy-clean image

123 pairs and realistic noise model (Guo et al., 2019). To further provide an interactive strategy to

124 conveniently correct the denoising results, the noise estimation subnetwork with asymmetric

125 learning was embedded in CBDNet to suppress the underestimation of the noise level. Couturier

126 et al. applied the deep encoder-decoder network (EDNet) to address additive white Gaussian and

127 multiplicative speckle noises (Couturier et al., 2018). The encoder module used to extract

128 features and remove the noise, whereas the decoder module recovered a clean image. To yield a

129 performance improvement, there are some methods using generative adversarial network (GAN)

130 in the training phase. Lsaiari et al. performed image denoising using generative adversarial

131 network (GAN) (Lsaiari et al. 2019). Yang et al. introduced a new CT image denoising method

132 based on GAN with Wasserstein distance and perceptual similarity (Yang et al. 2018). Dong et

133 al. developed a custom GAN to denoise optical coherence tomography (Dong et al. 2020). Lee et

134 al. proposed a model consisting of multiple U-Nets (MuNet) for three-dimensional neural image

135 denoising (Lee et al., 2020). It consisted of multiple U-Nets and using GAN in the training

136 phase. These methods perform well in removing Gaussian noise, but they cannot accurately

137 suppress speckle noise. Wang et al. proposed a set of convolutional layers along with a

138 componentwise division residual layer and a rectified linear unit (ReLU) activation function and 
139 BN to remove speckle noise (Wang et al., 2017). However, such a method cannot deal with the

140 speckle noise of ultrasound images well.

141 In this thesis, we proposed a generative adversarial network with residual dense connectivity

142 and weighted joint loss (GAN-RW) to overcome the limitations of traditional image denoising

143 methods and surpass the most advanced performance of ultrasound image denoising. The

144 proposed network consists of a denoising network and a discriminator network. The denoising

145 network is based on U-Net architecture which includes four encoder and four decoder modules.

146 Each block of the encoder and decoder is replaced with residual dense connectivity and BN to

147 remove speckle noise. The discriminator network applies a series of convolutional layers to

148 identify differences between the translated images and the desired modality. In the training

149 processes, we introduced a joint loss function consisting of a weighted sum of the L1 loss, the

150 perceptual loss function and the binary cross-entropy with logit loss (BCEWithLogitsLoss)

151 function. Experiments on natural images and ultrasound images illustrate that the proposed

152 algorithm surpasses the deep learning-based algorithms and conventional denoising algorithms.

153 The rest of the paper is organized as follows. Section 2 provides the proposed method and

154 implementation details. Extensive experiments are conducted to evaluate our proposed methods

155 in Section 3. We discuss these results in Section 4 and conclude in Section 5.

156 Materials \& Methods

157 An overview of the proposed network framework for ultrasound image denoising is shown in

158 Figure 1. In this section, the network architecture is introduced in detail.

159

160 Speckle Noise Model 
161 Due to speckle noise, ultrasound image processing is a very challenging task. Speckle noise is an

162 interference mode generated by the coherent accumulation of random scattering in the ultrasonic

163 beam resolution element, so it has the characteristics of asymmetrical intensity distribution and

164 significant spatial correlation (Slabaugh et al., 2006). This characteristic has an adverse effect on

165 the image quality and interpretability. Because these characteristics are difficult to model, many

166 methods of ultrasound image processing only assume that speckle noise is Gaussian noise,

167 resulting in these speckle noise models are more suitable for X-ray and MRI image than

168 ultrasound image. The gamma distribution (Sarti et al., 2005) and Fisher-Tippett distribution

169 (Michailovich et al., 2003) have been proposed to approximate speckle noise. Slabaugh G et al.

170 argued that Fisher-Tippett distribution was suitable for fully formed speckle noise in the

171 ultrasound image (Slabaugh et al., 2006). In this article, the speckle noise model of the

172 ultrasound image is given as:

$173 v(x, y)=u(x, y)+u(x, y)^{r} \theta(x, y)$

174 where $v(x, y)$ is the pixel location of the speckle noise image, $u(x, y)$ is the pixel location of the

175 noise-free ultrasound image, $\theta(x, y)$ is additive white Gaussian noise (AWGN) with zero-mean

176 and variance $\sigma^{2}$, and $r$ is associated with ultrasonic equipment. A large number of studies have

177 shown that $r=0.5$ is the best value that can be used to simulate speckle noise in ultrasonic images

178 (Yu et al., 2018; Lan et al., 2020).

179

180 Denoising Network

181 The architecture of denoising network is shown in Figure 2 (a). The denoising network is

182 based on U-Net, which consists of a contracting path and an expanding path. The expanding

183 function of the decoder module is to gradually restore the spatial and boundary information. The 
184 contracting function of the encoder module is to gradually reduce the spatial dimensions and 185 capture high-level feature information. Nevertheless, these successive convolutions and pooling 186 layers cause the loss of spatial information. Additionally, the problem of vanishing gradient is a 187 key point that hinders the networks from training as the networks deepen. Some densely 188 connected methods capture more information and avoid the appearance of vanishing-gradient 189 problem (Huang et al.,2017; Zhang et al., 2018; Park et al.,2019). Inspired by these methods, 190 we applied two residual dense connectivity blocks (RDCBs) to each module of the encoder and 191 decoder modules. The architecture of RDCBs is shown in Figure 2 (b). The RDCBs is composed 192 of three convolutional layers followed by BN and ReLU. Each module applies the previous 193 feature map through dense connectivity. We adopt dense connectivity and local residual learning 194 to improve the information flow so that the proposed algorithm can avoid the vanishing gradient 195 problem and accurately remove speckle noise. Meanwhile, RDCBs can capture more features to 196 improve denoising performances.

197 The network architecture of the encoder module is shown in Figure 2 (c). The encoder 198 module is composed of two RDCBs, a downsampling module and a convolution module. The 199 downsampling module is a $2 \times 2$ max-pooling layer. The convolutional module is a $1 \times 1$ 200 convolution layer followed by BN and ReLU. The feature map is fed into two RDCBs to 201 preserve more feature information and avoid vanishing gradient. Subsequently, the feature map 202 is fed into $2 \times 2$ max-pooling layers decreasing the size of feature map. Finally, the feature map is 203 fed into a $1 \times 1$ convolution layer followed by BN and ReLU. The size of the output feature maps 204 of the encoder module is half the size of the input feature maps.

205 The architecture of the decoder module is shown in Figure 2 (d). It is the inverse process of 206 the encoder module. It consists of three modules: two RDCBs, a $1 \times 1$ convolution layer followed 
207 by $\mathrm{BN}$ and ReLU and a subpixel interpolation layer. We use a $1 \times 1$ convolution layer to refine the 208 feature maps. Compared with the $2 \times 2$ deconvolution layer, subpixel interpolation can expand the 209 feature maps size more accurately and efficiently. Therefore, the size of the output feature map 210 of the upsampling block is twice the size of the input feature map, and the number of channels of 211 the input feature map is one second.

\section{Discriminator}

214 The discriminator is trained to distinguish the difference between the denoising image and 215 the standard image, where the denoising attempts to fool the discriminator. It uses a set of 216 convolutional layers to build a discriminative network. It consists of an input convolutional layer 217 and nine convolutional layers followed by BN and ReLU. The output channels of consecutive 218 convolutional layers are $64,128,256,512$ and 1 . Therefore, when the input image is passed 219 through each convolution block, the spatial dimension is decreased by a factor of two. The 220 architecture of the discriminator network framework for ultrasound image denoising is shown in 221 Figure 3.

222

\section{Loss Function}

224 Traditionally, learning-based image restoration uses the per-pixel loss between the restored 225 image and ground truth as the optimization target, and excellent quantitative scores can be 226 obtained. Nevertheless, in recent studies, relying only on low-level pixels to minimize pixelwise 227 errors has proven that it can lead to the loss of details and smooth the results (Johnson et al., 228 2016). In this paper, we use a weighted sum of the loss function. It consists of the denoising loss, 229 the perceptual loss of the feature extractor and the discriminator loss. 
231 differences between the standard image and the denoising image. The L1 loss is used and

232 calculated as follows:

$233 L 1=\sum_{i=1}^{n}|x-y|$

234 where $x$ is the denoising image and $y$ is the corresponding ground truth.

235 Recent studies have shown that the target image and the output image have similar feature 236 representations, not just every low-level pixel that matches them (Johnson et al., 2016). The

237 critical point is that the pretrained convolutional neural model used for image semantic

238 segmentation or classification has learned to encode image features, and these features can be 239 directly used for perceptual loss.

240 To preserve image details more effectively in removing noise, we use perceptual loss as one 241 of the loss functions, which is calculated by:

$242 L_{\text {per }}=\left\|\theta\left(y_{\text {true }}\right)-\theta\left(y_{\text {out }}\right)\right\|_{2}^{2}$

243 Where $\theta$ represents the feature extraction operator of the pretrained network. The convolution

244 neural network pre-trained for image classification which has already learned to capture features.

245 These features can be used as perceptual loss. In our proposed method, we adopt the output

246 before the first pooling layer from the pretrained VGG-19 network to extract features as

247 perceptual loss (Gong et al., 2018). To the discriminator network, we use BCEWithLogitsLoss to 248 discern the output image quality from the denoising network and the standard image. Then, we 249 obtain the weighted joint loss function, which consists of L1 loss $(L 1)$, perceptual loss (Lper) and 250 BCEWithLogitsLoss $\left(L_{B C E}\right) . \lambda_{1}, \lambda_{2}, \lambda_{3}$ are scalar weights for $L_{\text {loss }}$.

$251 L_{\text {loss }}=\lambda_{1} \mathrm{~L}_{1}+\lambda_{2} \mathrm{~L}_{\text {per }}+\lambda_{3} \mathrm{~L}_{\mathrm{BCE}}$

$252 \lambda_{1}=1, \lambda_{2}=0.1, \lambda_{3}=1$ 


\section{Training and Testing details}

255 To train our network, we use the Berkeley segmentation dataset (BSD400) composed of 400 256 images of size $180 \times 180$ for training (Martin et al., 2001; Zhang et al., 2017; Chen et al., 2016).

257 Then, according to Equation (1), speckle noise is added to the datasets and the noisy images are

258 generated. For training data that have three noise levels, we train the model for speckle denoising 259 with noise levels $\sigma=15,25$ and 50 independently. We set the patch sizes to $40 \times 40$ to train our 260 model. To avoid overfitting, we apply data augmentation by randomly rotating and flipping. The 261 initial learning rate is set to 1e-4 and halved every 2000 epochs. We use Adam optimizer and a 262 batch size of 32 during training.

263 For the test images, we adopt Berkeley segmentation (BSD68) (Martin et al,.2001; Roth et $264 a l ., 2009$ ) datasets for grey synthetic noisy images, which include 68 natural images, $321 \times 481$ or $265481 \times 321$ in size. To further verify the practicality of the proposed GAN-RW method, we also 266 illustrate the results of our method as well as eight existing denoising methods for ultrasound 267 images from the Kaggle Challenge (Rebetez et al., 2016), the Grand Challenge (Thomas et al., 268 2018) and lymph node datasets (Zhang et al., 2009). We applied PyTorch (version 1.7.0) as the 269 framework to implement our network. Training takes place on a workstation equipped with an 270 NVIDIA 2080Ti graphic card with 11 GB of memory.

272 seed points will get different results. Therefore, we repeated each training three times with the 273 same parameters and seeds, and then used the results of three experiments on test datasets to 274 obtain the mean value and standard deviation. 


\section{Evaluation Metrics}

277 In order to test the performance of the proposed method, the peak signal-to-noise ratio 278 (PSNR) (Chan et al., 1983) and the structural similarity (SSIM) (Wang et al., 2004) are used to 279 verify quantitative metrics. Meanwhile, the denoising results are used to show the visual quality 280 of denoising images. If the denoising method has higher the PSNR and SSIM results on the test 281 datasets, the denoising network shows better performance. In addition, to clarify the visual effect 282 on the denoised images, we zoom in on the area of the denoising image for display. If the 283 magnified area is clearer, it shows that the denoising method is more effective than others.

\section{Results}

To demonstrate the superiority of our proposed method in despeckling effect, we compared 286 our proposed network with deep learning-based methods and traditional denoising algorithms.

The methods for these comparisons were as follows: BM3D (Dabov et al., 2007), DnCNN (Zhang et al., 2017), DnCNN_Enhanced (Jifara et al., 2019), BRDNet (Tian et al., 2020), DHDN (Park et al., 2019), CBDNet (Guo et al., 2019), MuNet (Lee et al., 2020) and EDNet (Couturier et al., 2018). Two performance metrics are used, namely, PSNR and SSIM, which are expressed in terms of average value and standard deviation. Statistical analysis was performed with SPSS statistics software (version 26.0; IBM Inc., Armonk, NY, USA). All deep-learning based methods were trained three times and BM3D used three different parameters to obtain the average value and standard deviation. Experiments were performed on the BSD68 and ultrasound images.

\section{The BSD68}

Table I shows the mean, standard deviation of the proposed methods and the compared 
299 noise level was 15, the average PSNR and SSIM of our proposed method improved by $1.21 \mathrm{~dB}$

300 and 0.0113 , which were better than those of the compared method. The average performance of

301 the GAN-RW increased by approximately $3.58 \%$ and $1.23 \%$ for PSNR and SSIM, respectively.

302 When the noise level was 25 , the average PSNR and SSIM of this method improved by $0.96 \mathrm{~dB}$

303 and 0.0160 , and increased by approximately $3.08 \%$ and $1.84 \%$ for PSNR and SSIM,

304 respectively. When the noise level was 50, the average PSNR and SSIM of this method

305 improved by $0.36 \mathrm{~dB}$ and 0.0156 and increased by approximately $1.32 \%$ and $1.98 \%$ for PSNR

306 and SSIM, respectively. As shown in Table I, the proposed method is superior to the traditional

307 methods for three noise levels.

308 To compare subjective performance, we compared the denoising images for different

309 methods. Figure 4, 5 and 6 show the grey scale denoising image of the proposed methods and the 310 compared method at different noise levels. To easily observe the performance of GAN-RW and

311 other methods, we zoomed in on an area from denoising images obtained using the compared

312 methods. In Figure 4, the proposed method accurately restored the pattern, while the compared

313 methods achieved blurred denoising image. As shown in Figure 5, the compared methods failed

314 to exactly restore the windows or achieved blurred denoising image. However, the proposed

315 method restored the windows accurately. Similarly, unlike the compared methods, the details of

316 the zebra stripes could not be restored. The proposed method restored the details in Figure 6. As

317 shown in these images under different noise levels, the traditional methods produced blurred

318 results and could not restore the details of the patterns, while the proposed method accurately

319 restored the patterns.

320

321 Ultrasound Images 
We used the ultrasound images of lymph nodes, the foetal head and the brachial plexus with

323

324

325

326

327

328

329

330

331

332

333

334

335

336

337

338

339

340

341 followed by BN and ReLU and each module applied the previous feature map through dense

342 connectivity. We used two successive convolutional layers followed by BN and ReLU without

343 dense connectivity (GAN-RW-WD) to replace two RDCBs. The experimental results compared

344 with GAN-RW are shown in Table I. When the noise level was 15, RDCBs can enhance the 
345 average PSNR by approximately $0.44 \%$ and the average SSIM by approximately $0.16 \%$ for the

346 BSD68, respectively. When the noise level was 25 , RDCBs can enhance the average PSNR by

347 approximately $0.53 \%$ and the average SSIM by approximately $0.57 \%$ for the BSD 68 ,

348 respectively. When the noise level was 50, RDCBs can enhance the average PSNR by

349 approximately $0.37 \%$ and the average SSIM by approximately $0.61 \%$ for the BSD68,

350 respectively.

351

352 Statistical Analysis

353 Statistical analysis is necessary to verify the superiority of the proposed method. Due to the

354 PSNR and SSIM values were not Gaussian distribution, we used the nonparametric Friedman

355 test (Friedman., 1937) to assess the performance of different denoising algorithms. The mean

356 rank and p-Value of PSNR and SSIM of all algorithms are shown in Table II. Usually, a p-value

357 of less than 0.05 is deemed the significant difference. The mean rank presents the performance of

358 different algorithms, and the higher value of mean rank has the better performance. It can be seen

359 from Table II that GAN-RW has a significant improvement over other algorithm.

360

361 Discussion

362 In this paper, we proposed a generative adversarial network for ultrasound image despeckling.

363 The GAN-RW is based on U-Net with residual dense connectivity, BN and a joint loss function

364 to remove speckle noise. We used natural images and ultrasound images to verify our method.

365 For the BSD68 test datasets, when the noise level was 15, our method achieved $35.28 \mathrm{~dB}$ and

3660.9404 for PSNR and SSIM. Compared with the original noise image, the average values of the

367 GAN-RW increased by approximately $10.05 \%$ and $12.26 \%$ for PSNR and SSIM, respectively. 
368 When the noise level was 25 , our method achieved $32.15 \mathrm{~dB}$ and 0.8969 for PSNR and SSIM,

369 respectively. Compared with the original noise image, the average performance of the GAN-RW

370 increased by approximately $16.01 \%$ and $28.43 \%$ for PSNR and SSIM. When the noise level was

371 50, our method achieved 27.74dB and 0.8064 for PSNR and SSIM, respectively. Compared with

372 the original noise image, the average performance of the GAN-RW increased by approximately

$37326.88 \%$ and $75.35 \%$ for PSNR and SSIM, respectively. In Figure 10, boxplots show the

374 comparison of PSNR and SSIM under different noise levels for BSD68. In the end, we used the

375 ultrasound images of lymph nodes, the brachial plexus and the foetal head to verify the

376 practicality of the proposed GAN-RW. In contrast, GAN-RW can effectively eliminate speckle

377 noise while retaining image details better and improving the visual effect.

378

379 Conclusions

380 In conclusion, we developed and verified a new ultrasound image despeckling method. GAN-

$381 \mathrm{RW}$ is based on U-Net and uses residual dense connectivity, $\mathrm{BN}$ and joint loss functions to

382 remove speckle noise. Compared with BM3D, DnCNN, DnCNN-Enhanced, BRDNet, DHDN,

383 CBDNet, MuNet, EDNet and GAN-RW achieves better despeckling performance on three fixed

384 noise levels of BSD68. We also effectively verified the proposed method on ultrasound images

385 of lymph nodes, the brachial plexus and the foetal head.

386

387 Acknowledgements

388 We would like to thank Bo Li and Yang Zhao for their constructive discussion during the

389 manuscript revision. 


\section{Data Availability}

392 The following information was supplied regarding data availability:

393 Datasets of the BSD400, BSD 68 and lymph node is available at GitHub:

394 https://github.com/smartboy110/denoising-datasets

395 Datasets of the foetal head is available at: https://zenodo.org/record/1327317\#.YXyyqbG_9ZE

396 Datasets of the brachial plexus is available at: https://www.kaggle.com/c/ultrasound-nerve-

397 segmentation

398

399

References

400

401

Abedalla A, Abdullah M, Al-Ayyoub M, Benkhelifa E. 2021. Chest X-ray pneumothorax

402

segmentation using U-Net with EfficientNet and ResNet architectures. PeerJ Computer Science.

403

7: e607.

404 Buades A, Coll B, Morel J M. 2005. A non-local algorithm for image denoising. Proceedings of 405 the IEEE conference on computer vision and pattern recognition (CVPR). 2: 60-65.

406 Chan L C, Whiteman P. 1983. Hardware-constrained hybrid coding of video imagery. IEEE

407 Transactions on Aerospace and Electronic Systems. (1): 71-84.

408 Couturier R, Perrot G, Salomon M. 2018. Image denoising using a deep encoder-decoder

409 network with skip connections. International Conference on Neural Information Processing.

$410 \quad 554-565$.

411 Dabov K, Foi A, Katkovnik V, Egiazarian K. 2007. Image denoising by sparse 3-D transform-

412 domain collaborative filtering. IEEE Transactions on image processing. 16(8): 2080-2095. 
413 Dong Z, Liu G, Ni G, Jerwick J, Duan L, Zhou C. 2020. Optical coherence tomography image

414 denoising using a generative adversarial network with speckle modulation. Journal of

415 biophotonics. 13(4): e201960135.

416 Fawakherji M, Youssef A, Bloisi D, Pretto A, Nardi D. 2019. Crop and weeds classification

417 for precision agriculture using context-independent pixel-wise segmentation. Third IEEE

418 International Conference on Robotic Computing (IRC). 146-152.

419 Friedman M. 1937. The Use of Ranks to Avoid the Assumption of Normality Implicit in the 420 Analysis of Variance. Journal of the American Statistical Association.32(200): 675-701.

421 Frost V S, Stiles J A, Shanmugan K S, Holtzman J C. 1982. A model for radar images and its 422 application to adaptive digital filtering of multiplicative noise. IEEE Transactions on pattern 423 analysis and machine intelligence. 2: 157-166.

424 Gong K, Guan J, Liu C C, Qi J. 2018. PET Image Denoising Using a Deep Neural Network 425 Through Fine Tuning. IEEE Transactions on Radiation and Plasma Medical. 3(2): 153-161. 426 Guo S, Ya Z, Zhang K, Zuo W, Zhang L. 2019. Toward convolutional blind denoising of real 427 photographs. Proceedings of the IEEE conference on computer vision and pattern recognition 428 (CVPR).1712-1722.

429 Gupta S, Chauhan R C, Sexana S C. 2004. Wavelet-based statistical approach for speckle 430 reduction in medical ultrasound images. Medical and Biological Engineering and 431 computing. 42(2): 189-192.

432 He K, Zhang X, Ren S, Sun J. 2016. Deep residual learning for image recognition. Proceedings 433 of the IEEE conference on computer vision and pattern recognition (CVPR). 770-778. 
434 Huang G, Liu Z, Van Der Maaten L, Weinberger K Q. 2017. Densely connected

435 convolutional networks. Proceedings of the IEEE conference on computer vision and pattern 436 recognition (CVPR). 4700-4708.

437 Isaiari A, Rustagi R, Thomas M M, Forbes A G. 2019. Image denoising using a generative 438 adversarial network. 2019 IEEE 2nd International Conference on Information and Computer 439 Technologies (ICICT). 126-132.

440 Jaiswal A, Upadhyay J, Somkuwar A. 2014. Image denoising and quality measurements by 441 using filtering and wavelet based techniques. AEU-International Journal of Electronics and 442 Communications. 68(8): 699-705.

443 Jifara W, Jiang F, Rho S, Cheng M, Liu S. 2019. Medical image denoising using 444 convolutional neural network: a residual learning approach. The Journal of Supercomputing. 445 75(2): 704-718.

446 Johnson J, Alahi A, Li F F. 2016. Perceptual losses for real-time style transfer and super447 resolution. European conference on computer vision. 694-711.

448 Kuan D, Sawchuk A, Strand T, Chavel P. 1987. Adaptive restoration of images with speckle. 449 IEEE Trans on Acoust, Speech and Signal Process. 35(3): 373-383.

450 Lan Y, Zhang X. 2020. Real-time ultrasound image despeckling using mixed-attention 451 mechanism based residual UNet. IEEE Access. 8: 195327-195340.

452 Lee S, Negishi M, Urakubo H, Kasai H, Ishii S. 2020. Mu-net: Multi-scale U-net for two453 photon microscopy image denoising and restoration. Neural Network. 15: 92-103.

454 Martin D, Fowlkes C, Tal D, Malik J et al. 2001. A database of human segmented natural 455 images and its application to evaluating segmentation algorithms and measuring ecological 456 statistics. International Conference on Computer Vision. 416-423. 
457 Michailovich O, Adam D. 2003. Robust Estimation of Ultrasound Pulses Using Outlier-

458 Resistant De-Noising. IEEE Trans. on Medical Imaging. 22(3): 368-392.

459 Negi A, Raj A N J, Nersisson R, Zhuang Z, Murugappan M. 2020. RDA-UNET-WGAN: an

460 accurate breast ultrasound lesion segmentation using wasserstein generative adversarial

461 networks. Arabian Journal for Science and Engineering. 45(8): 6399-6410.

462 Park B, Yu S, Jeong J. 2019. Densely connected hierarchical network for image denoising.

463 Proceedings of the IEEE conference on computer vision and pattern recognition (CVPR).

464 Raj A N J, Zhu H, Khan A, Zhuang Z, Yang Z, Mahesh V G, Karthik G. (2021). ADID-

465 UNET-a segmentation model for COVID-19 infection from lung CT scans. PeerJ Computer

466 Science. 7: e349.

467 Rebetez J. 2016. Kaggle Ultrasound Nerve Segmentation. Available at

468 http://fhtagn.net/prog/2016/08/19/kaggle-uns.html\#dat

469 Ronneberger O, Fischer P, Brox T. 2015. U-net: Convolutional networks for biomedical image

470 segmentation. International Conference on Medical image computing and computer-assisted

471 intervention. 234-241.

472 Roth S, Black M J. 2009. Fields of experts. International Journal of Computer Vision. 82(2):

$473 \quad 205-229$.

474 Salimans T, Kingma D P. 2016. Weight normalization: a simple reparameterization to

475 accelerate training of deep neural networks. Advances in neural information processing systems

476 (NIPS). 29: 901-909.

477 Sarti A, Corsi C, Mazzini E, Lamberti C. 2005. Maximum Likelihood Segmentation of

478 Ultrasound Images with Rayleigh Distribution. IEEE Trans. on Ultrasonics, Ferroelectrics, and

479 Freq. Control, 52(6): 947-960.

Peer] Comput. Sci. reviewing PDF | (CS-2021:10:67114:2:0:NEW 5 Jan 2022) 
480 Shih A C C, Liao H Y M, Lu C S. 2003. A new iterated two-band diffusion equation: theory 481 and its application. IEEE Transactions on Image Processing. 12(4): 466-476.

482 Singh P, Mukundan R, de Ryke R. 2017. Synthetic models of ultrasound image formation for 483 speckle noise simulation and analysis. 2017 International Conference on Signals and System 484 (ICSigSys). IEEE: 278-284.

485 Slabaugh G, Unal G, Fang T, Wels M. 2006. Ultrasound-specific segmentation via 486 decorrelation and statistical region-based active contours. Proceedings of the IEEE conference 487 on computer vision and pattern recognition (CVPR). 1: 45-53.

488 Srivastava M, Anderson C L, Freed J H. 2016. A new wavelet denoising method for selecting 489 decomposition levels and noise thresholds. IEEE Access. 4: 3862-3877.

490 Thomas L A, van den Heuvel, Dagmar de Bruijn, Chris L. de Korte and Bram van 491 Ginneken. 2018. Automated measurement of fetal head circumference using 2D ultrasound 492 images [Data set]. Zenodo. http://doi.org/10.5281/zenodo.1322001

493 Tian C, Xu Y, Zuo W. 2020. Image denoising using deep CNN with batch renormalization. 494 Neural Networks. 121: 461-473.

495 Wang P, Zhang H, Patel V M. 2017. SAR image despeckling using a convolutional neural 496 network. IEEE Signal Processing Letters. 24(12): 1763-1767.

497 Wang Z, Bovik A C, Sheikh H R, Simoncelli E P. 2004. Image quality assessment: from error 498 visibility to structural similarity. IEEE transactions on image processing. 13(4): 600-612.

499 Chen Y, Pock T. 2016. Trainable nonlinear reaction diffusion: A flexible framework for fast and 500 effective image restoration. IEEE transactions on pattern analysis and machine intelligence.

501 39(6): 1256-1272. 
502 Yang Q, Yan P, Zhang Y, Yu, H, Shi, Y, Mou, X, Wang, G. 2018. Low-dose CT image

503 denoising using a generative adversarial network with Wasserstein distance and perceptual loss.

504 IEEE transactions on medical imaging. 37(6): 1348-135.

505 Yu H, Ding M, Zhang X, Wu J. 2017. PCANet based nonlocal means method for speckle noise 506 removal in ultrasound images. PLoS ONE. 13(10): e0205390.

507 Yu Y, Acton S T. 2002. Speckle reducing anisotropic diffusion. IEEE Transactions on image 508 processing. 11(11): 1260-1270.

509 Yue Y, Croitoru M M, Bidani A, Zwischenberger J B, Clark J W. 2006. Nonlinear

510 multiscale wavelet diffusion for speckle suppression and edge enhancement in ultrasound

511 images. IEEE Transaction on Medical Imaging. 25(3): 297-311.

512 Zhang J, Wang Y and Shi X. 2009. An improved graph cut segmentation method for cervical

513 lymph nodes on sonograms and its relationship with node's shape assessment. Computerized

514 Medical Imaging and Graphics. 33(8): 602-607.

515 Zhang K, Zuo W, Chen Y, Meng D, Zhang L. 2017. Beyond a Gaussian denoiser: residual

516 learning of deep CNN for image denoising. IEEE Transactions on image processing. 26(7):

$517 \quad 3142-3155$.

518 Zhang Lun, Zhang Junhua, Li Zonggui, Song Yingchao. 2020. A multiple-channel and 519 atrous convolution network for ultrasound image segmentation. Medical Physics. 47(12): 62705206285.

521 Zhang Y, Tian Y, Kong Y, Zhong B, and Fu Y. 2018. Residual dense network for image 522 restoration. arXiv preprint: arXiv:1812.10477. 
523 Zhou Z, Rahman Siddiquee M, Tajbakhsh N, Liang J. 2019. UNet++: A Nested U-Net

524 Architecture for Medical Image Segmentation. Deep Learn Med Image Anal Multimodal Learn

525 Clin Decis Support. 3-11.

526 Zhuang Z, Li N, Joseph Raj A N, Mahesh V G, Qiu S. 2019. An RDAU-NET model for

527 lesion segmentation in breast ultrasound images. PloS one. 14(8): e 0221535. 


\section{Figure 1}

Architecture of the proposed network.

The denoising network is tasked with translating input images to the target domain through encoder-decoder networks. The discriminator is trained to distinguish between standard and denoising images. The pre-trained VGG-19 is used to acquire more features as perceptual loss.

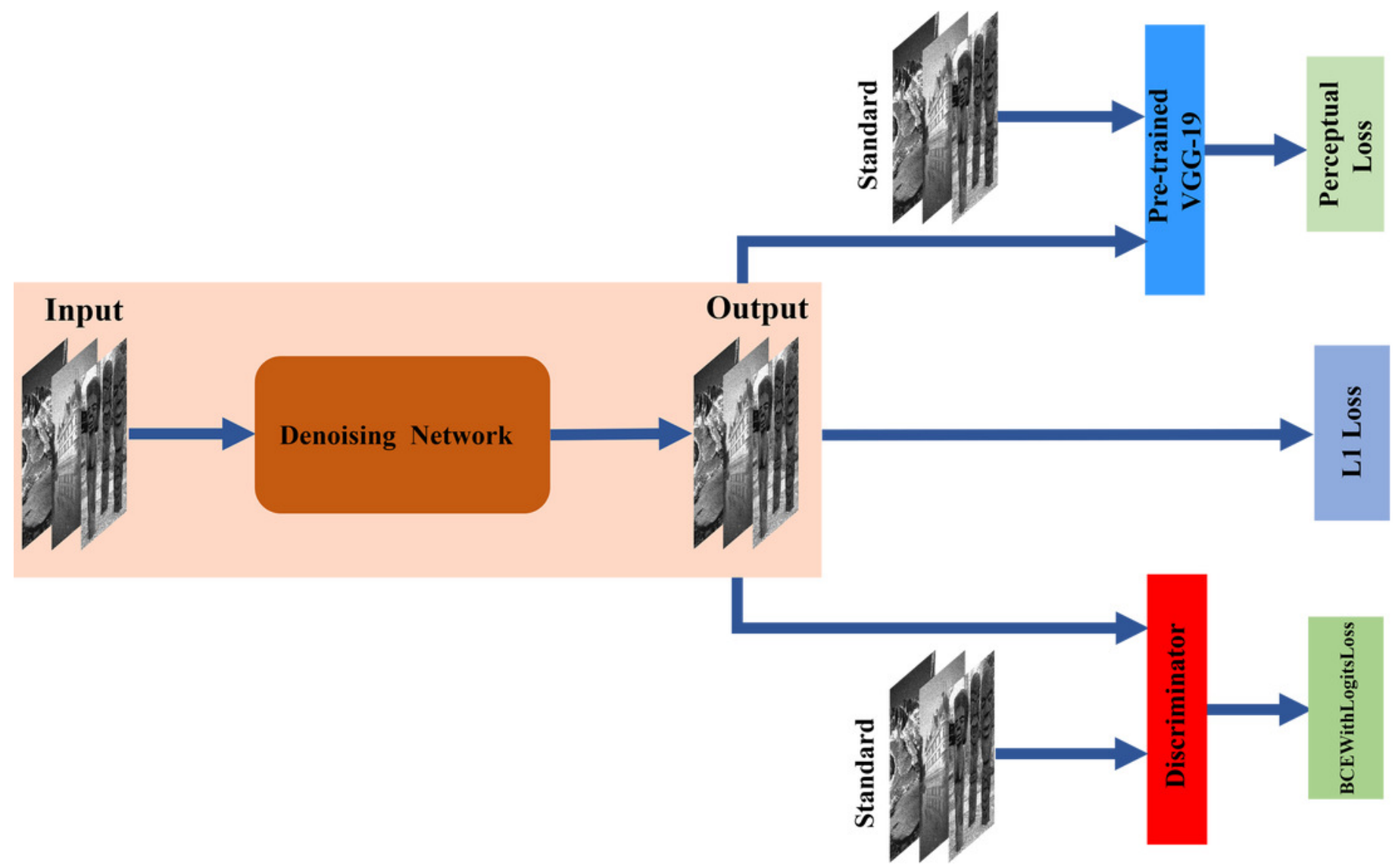


Figure 2

Architecture of the denoising network.

(a). Architecture of the denoising network. $\mathrm{H} \times \mathrm{W} \times \mathrm{C}$ specify the output dimensions of each component ( $C=1,128,256,512,1024)$. (b). Architecture of the RDCBs. (c). The architecture of the encoder module. (d). The architecture of the decoder module. Conv denotes a $3 \times 3$ convolution layer.

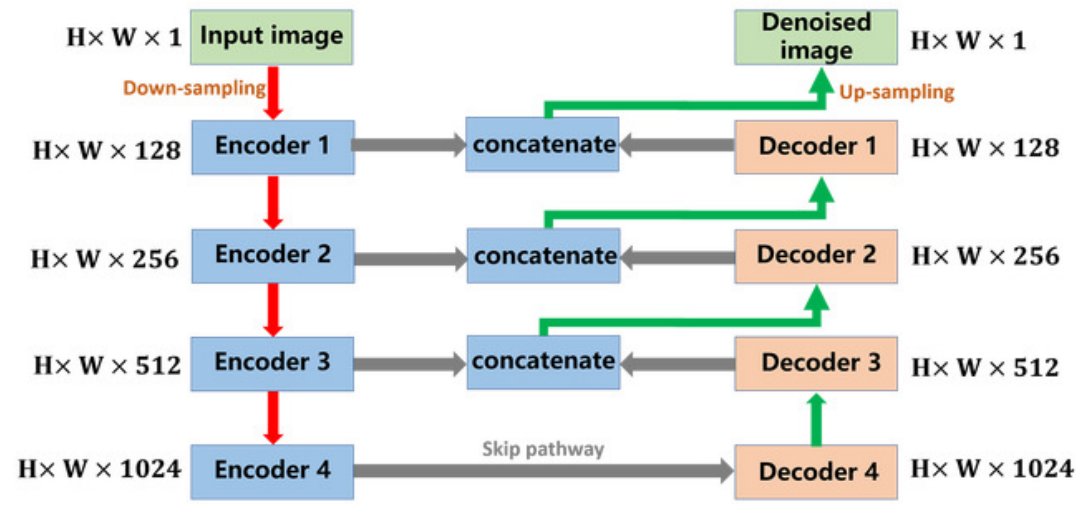

(a)

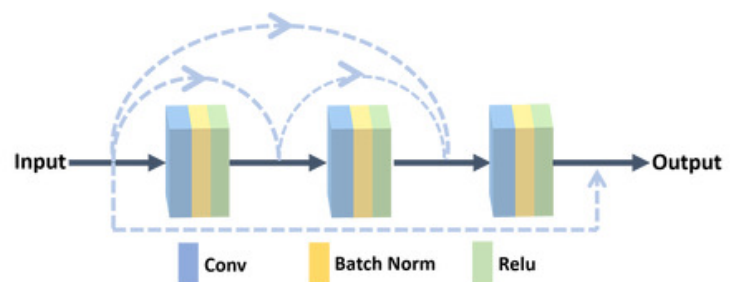

(b)

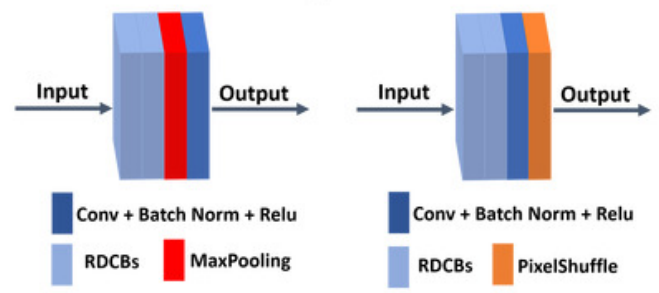

(c) 
Figure 3

The architecture of the discriminator network.

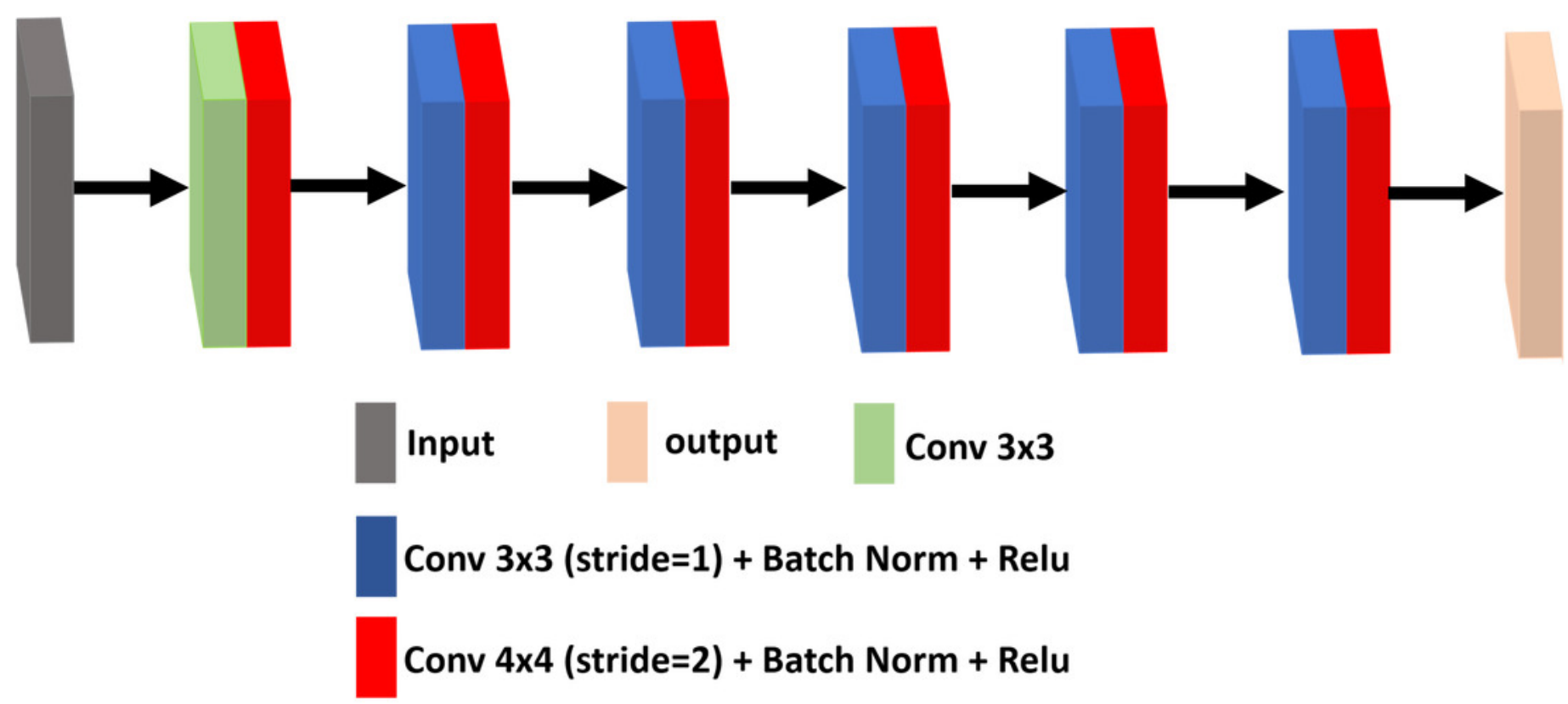


Figure 4

Speckle denoising results of the compared methods and the proposed method on noise level $\sigma=15$.

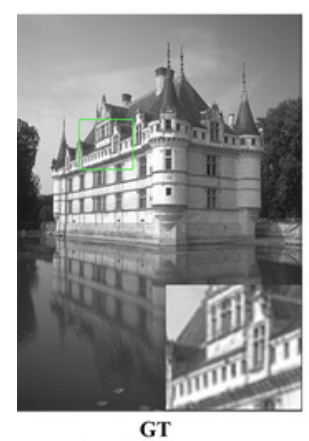

GT
(PSNR(dB)/SSIM)

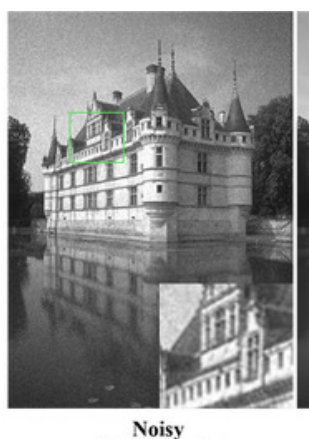

$(32.06 / 0.8377)$

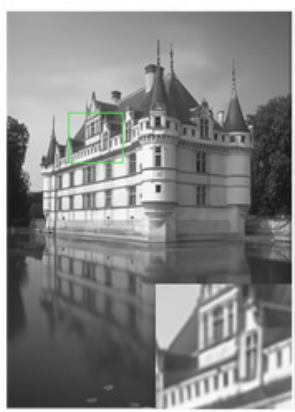

DHDN $(35.18 / 0.9393)$

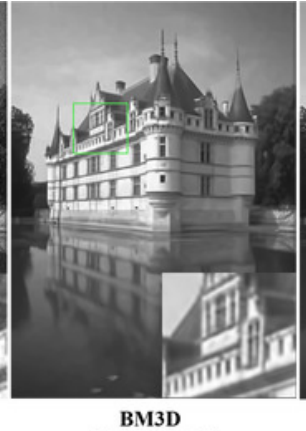

(33.30/0.9045)

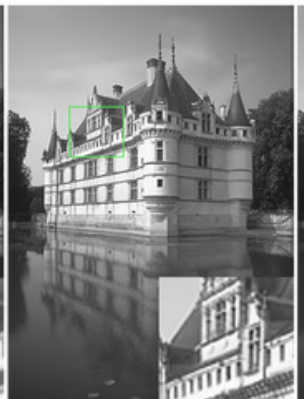

CBDNet

$(33.83 / 0.9334)$

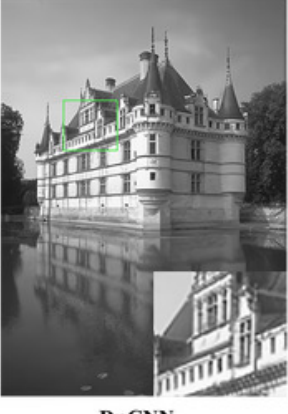

DnCNN

(33.86/0.9332)

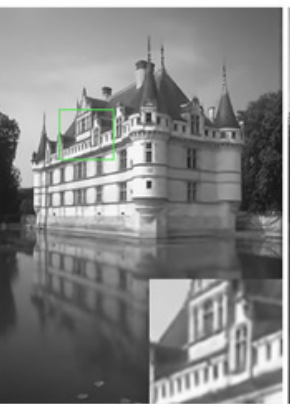

MuNet

$(34.67 / 0.9296)$

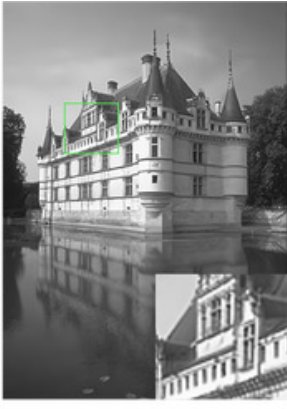

DnCNN-Enhanced

(33.87/0.9332)

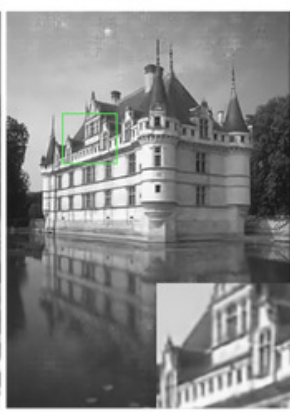

EDNet

$(34.07 / 0.9277)$
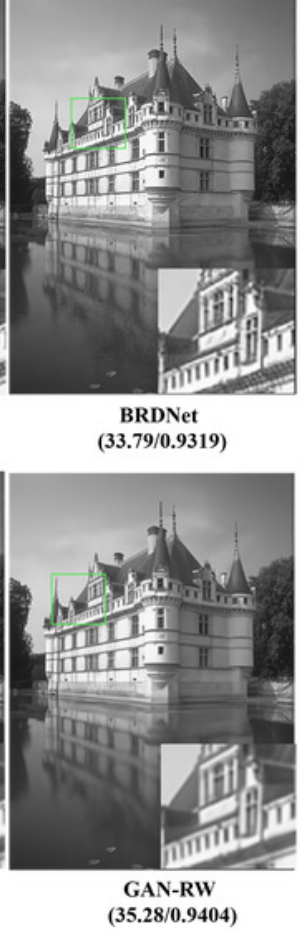
Figure 5

Speckle denoising results of the compared methods and the proposed method on noise level $\sigma=25$.
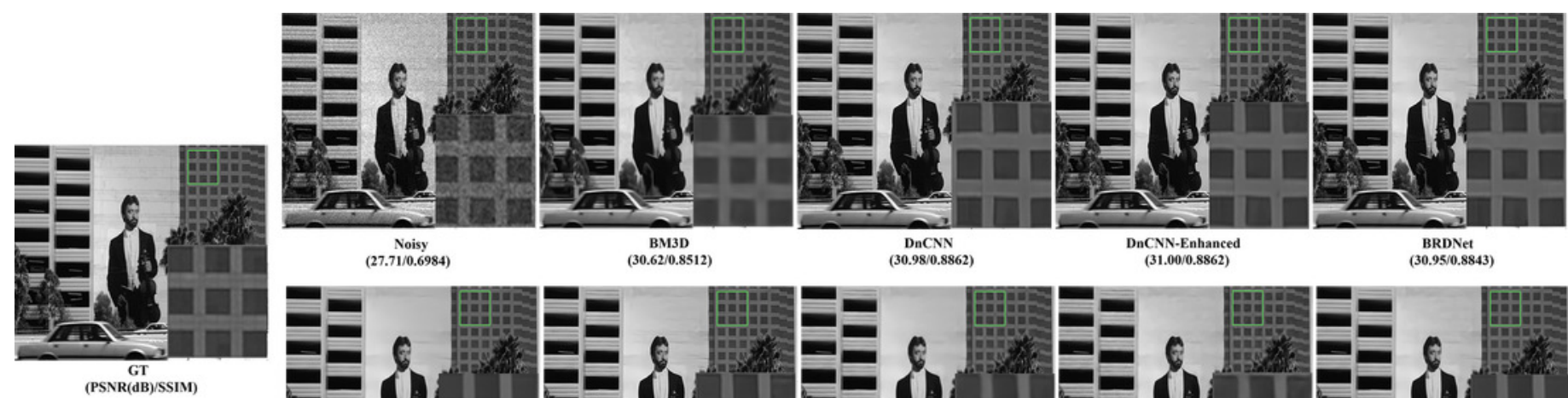

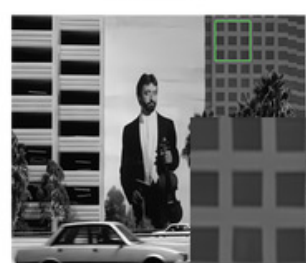

DHDN (32.03/0.8938

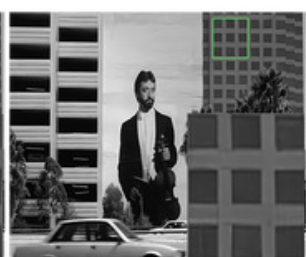
CBDNet
(31.01/0.8875

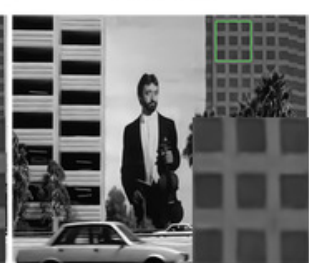

MuNet MuNet
(31.51/0.8780

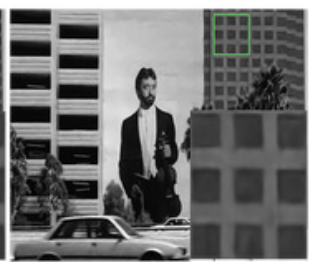

EDNet EDNet
(31.42/0.8799)

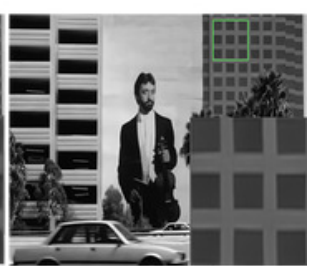

GAN-RW GAN-RW
$(32.15 / 0.8969)$ 


\section{Figure 6}

Speckle denoising results of the compared methods and the proposed method on noise level $\sigma=50$.

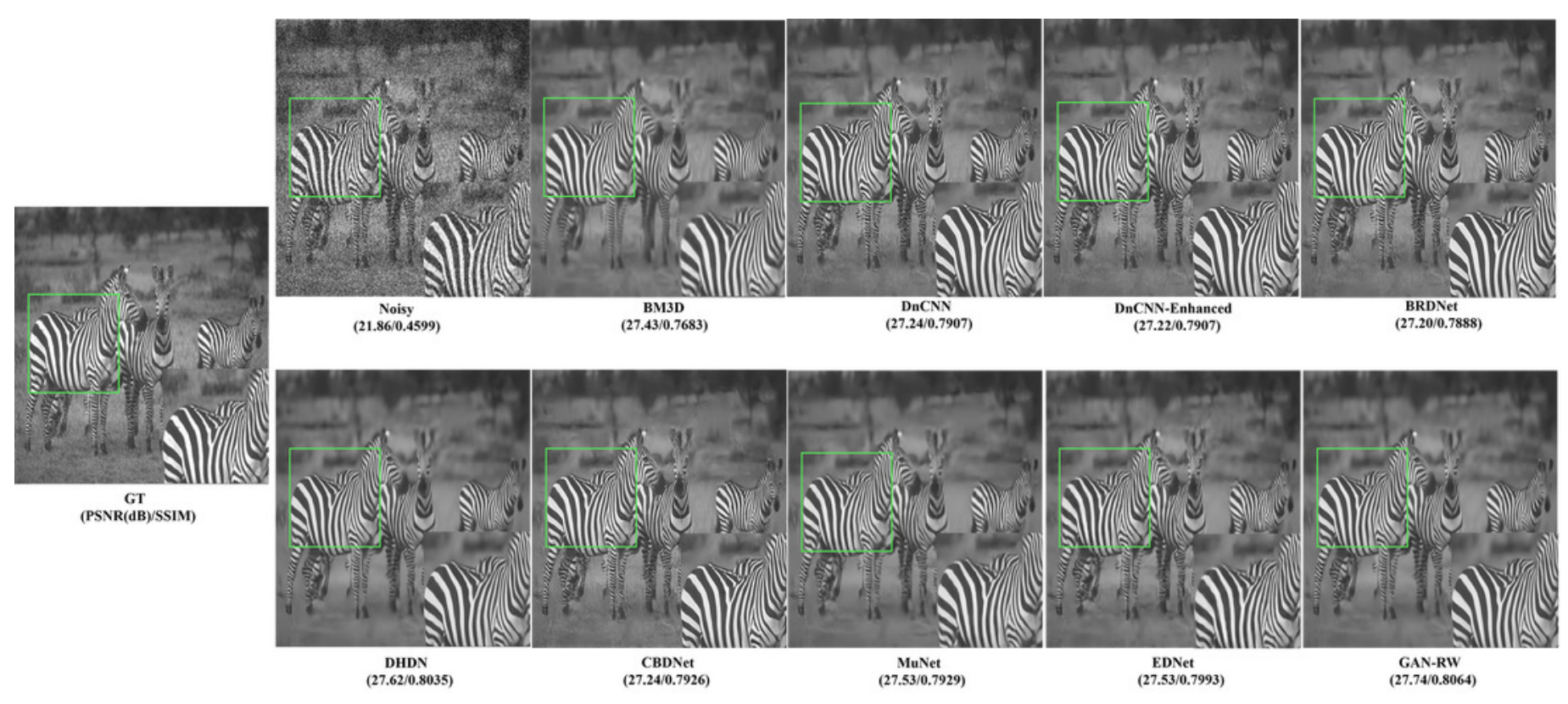




\section{Figure 7}

Speckle denoising results of the compared methods and the proposed method on the real ultrasound images of Lymph nodes.

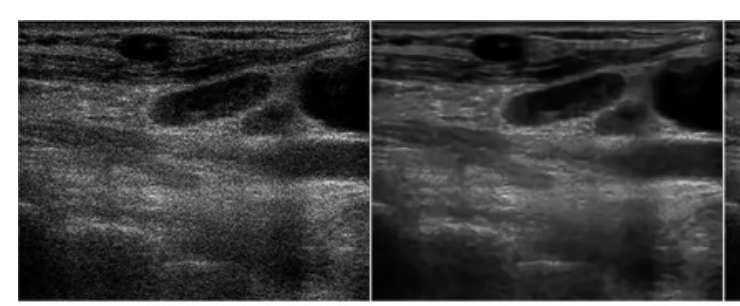

Noise

BM3D

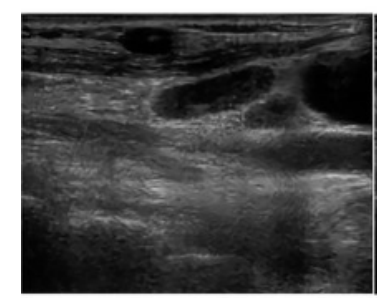

DHDN

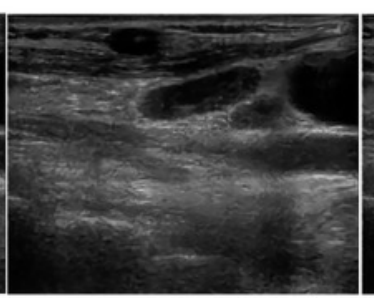

CBDNet

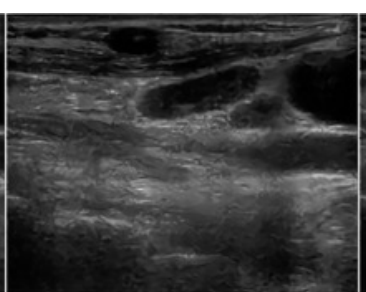

DnCNN

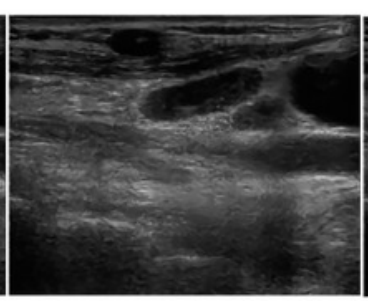

MuNet

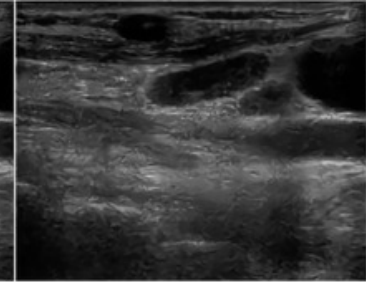

DnCNN-Enhanced

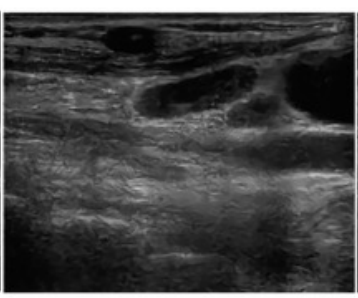

EDNet

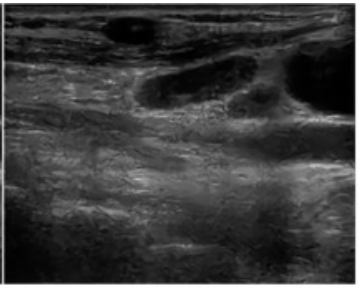

BRDNet

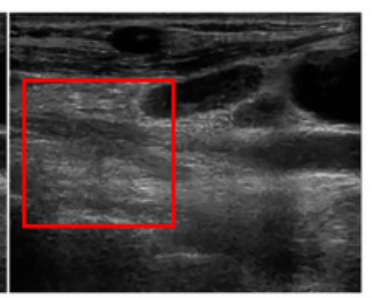

GAN-RW 


\section{Figure 8}

Speckle denoising results of the compared methods and the proposed method on the real ultrasound images of foetal head.

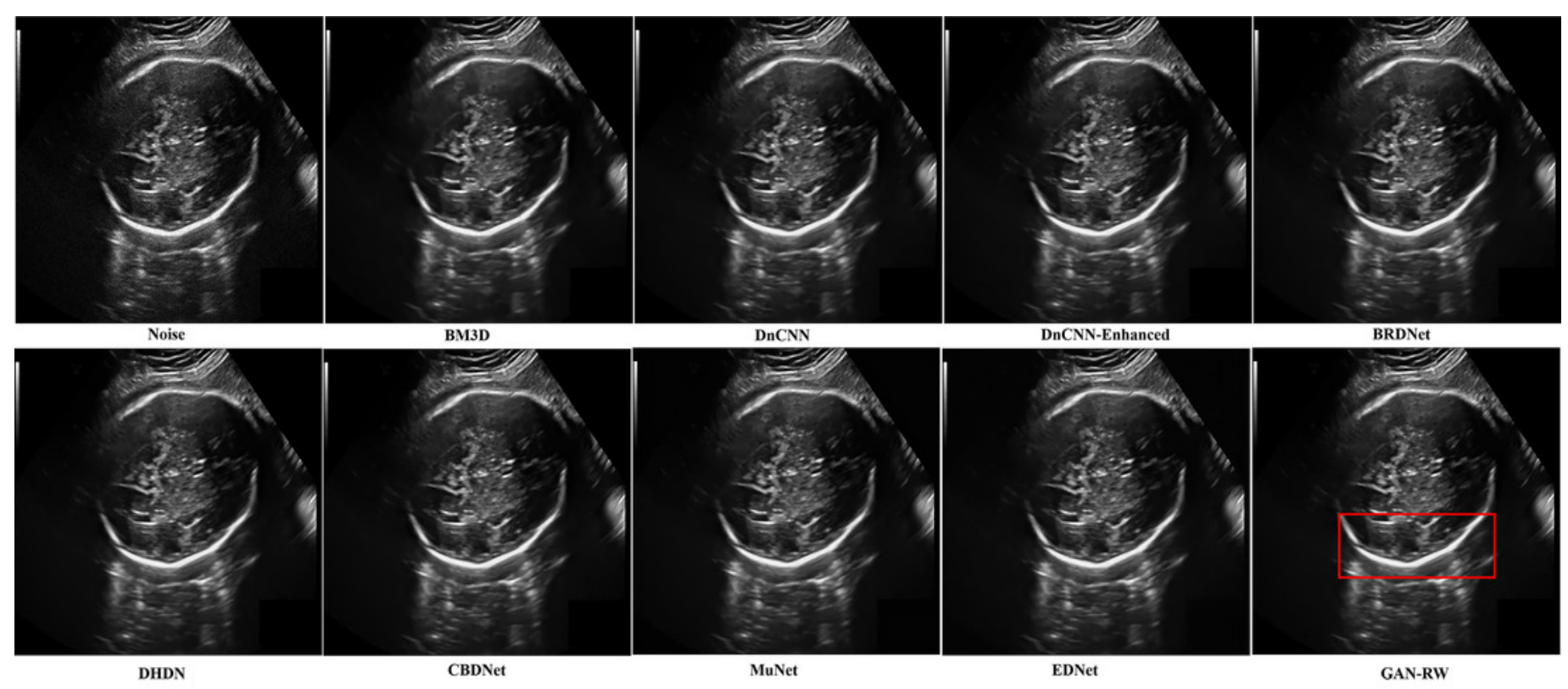




\section{Figure 9}

Speckle denoising results of the compared methods and the proposed method on the real ultrasound images of Brachia Plexus.
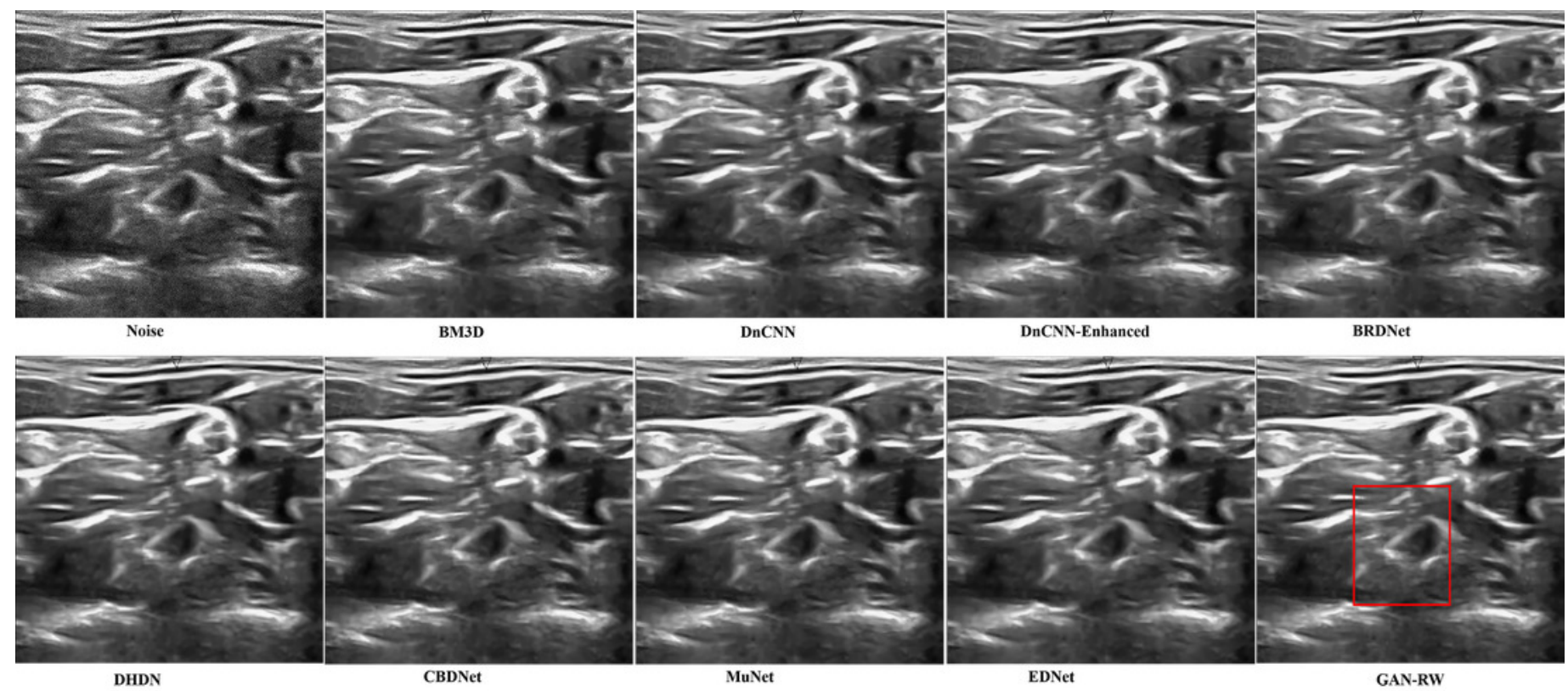
Figure 10

Boxplots of average PSNR ( $\mathrm{dB}$ ) and SSIM results of compared methods and proposed method for BSD68.
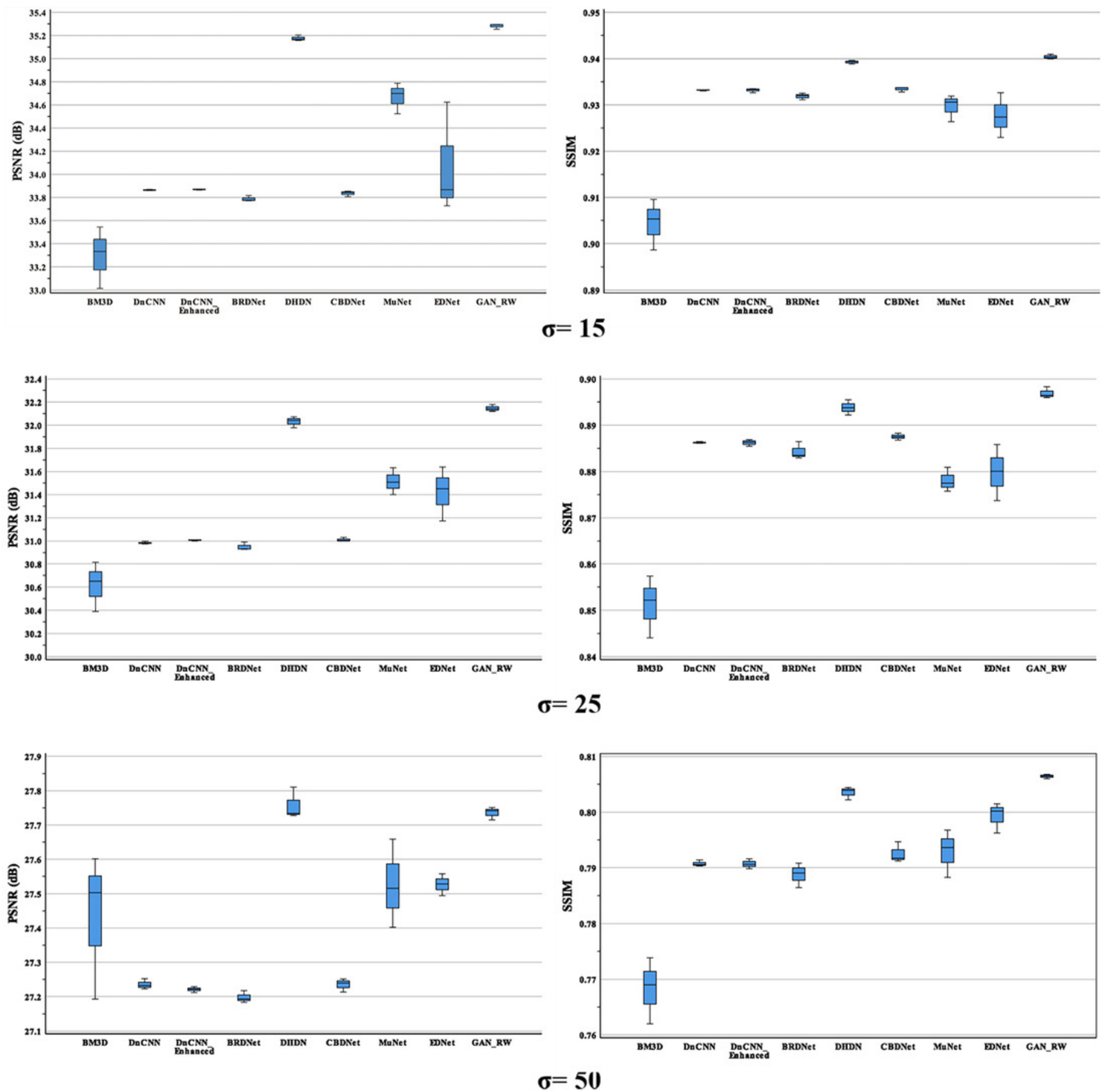


\section{Table $\mathbf{1}$ (on next page)}

The mean and standard deviation of the PSNR (dB) and SSIM values of different methods for denoising BSD68 gray images. The best result is highlighted with bold. 
1 Table I: The mean and standard deviation of the PSNR (dB) and SSIM values of different

2 methods for denoising BSD68 gray images. The best result is highlighted with bold.

\begin{tabular}{|c|c|c|c|c|c|c|}
\hline \multirow{3}{*}{ Method } & \multicolumn{6}{|c|}{ BSD68 } \\
\hline & \multicolumn{2}{|c|}{$\sigma=15$} & \multicolumn{2}{|c|}{$\sigma=25$} & \multicolumn{2}{|c|}{$\sigma=50$} \\
\hline & PSNR & SSIM & PSNR & SSIM & PSNR & SSIM \\
\hline Noisy & 32.06 & 0.8377 & 27.71 & 0.6984 & 21.86 & 0.4599 \\
\hline BM3D & $33.30 \pm 0.2178$ & $0.9045 \pm 0.0045$ & $30.62 \pm 0.1747$ & $0.8512 \pm 0.0055$ & $27.43 \pm 0.1737$ & $0.7683 \pm 0.0049$ \\
\hline DnCNN & $33.86 \pm 0.0043$ & $0.9332 \pm 0.0001$ & $30.98 \pm 0.0091$ & $0.8862 \pm 0.0002$ & $27.24 \pm 0.0124$ & $0.7907 \pm 0.0005$ \\
\hline DnCNN- Enhanced & $33.87 \pm 0.0036$ & $0.9332 \pm 0.0004$ & $31.00 \pm 0.0057$ & $0.8862 \pm 0.0006$ & $27.22 \pm 0.0066$ & $0.7907 \pm 0.0007$ \\
\hline BRDNet & $33.79 \pm 0.0202$ & $0.9319 \pm 0.0006$ & $30.95 \pm 0.0283$ & $0.8843 \pm 0.0015$ & $27.20 \pm 0.0140$ & $0.7888 \pm 0.0018$ \\
\hline DHDN & $35.18 \pm 0.0203$ & $0.9393 \pm 0.0003$ & $32.03 \pm 0.0388$ & $0.8938 \pm 0.0013$ & $27.62 \pm 0.0374$ & $0.8035 \pm 0.0009$ \\
\hline CBDNet & $33.83 \pm 0.0206$ & $0.9334 \pm 0.0004$ & $31.01 \pm 0.0145$ & $0.8875 \pm 0.0006$ & $27.24 \pm 0.0161$ & $0.7926 \pm 0.0015$ \\
\hline MuNet & $34.67 \pm 0.1099$ & $0.9296 \pm 0.0024$ & $31.51 \pm 0.0956$ & $0.8780 \pm 0.0022$ & $27.53 \pm 0.1049$ & $0.7929 \pm 0.0035$ \\
\hline EDNet & $34.07 \pm 0.3938$ & $0.9277 \pm 0.0039$ & $31.42 \pm 0.1916$ & $0.8799 \pm 0.0049$ & $27.53 \pm 0.0261$ & $0.7993 \pm 0.0022$ \\
\hline GAN-RW-WD & $35.13 \pm 0.0334$ & $0.9389 \pm 0.0003$ & $31.98 \pm 0.0378$ & $0.8919 \pm 0.0009$ & $27.63 \pm 0.0537$ & $0.8015 \pm 0.0028$ \\
\hline GAN_RW & $35.28 \pm 0.0193$ & $0.9404 \pm 0.0004$ & $32.15 \pm 0.0243$ & $0.8969 \pm 0.0010$ & $27.74 \pm 0.0151$ & $0.8064 \pm 0.0003$ \\
\hline
\end{tabular}

3

4

5

6

7

8

9

10

11

12

13

14

15

16

17

18

19

20

21

22 


\section{Table 2 (on next page)}

The mean rank (Friedman test) of the PSNR (dB) and SSIM values of the different methods for denoising BSD68 gray images. 
1 Table II: The mean rank (Friedman test) of the PSNR (dB) and SSIM values of the different

2 methods for denoising BSD68 gray images.

\begin{tabular}{|c|c|c|c|c|c|c|c|c|c|c|c|c|}
\hline \multirow{3}{*}{ Methods } & \multicolumn{4}{|c|}{$\sigma=15$} & \multicolumn{4}{|c|}{$\sigma=25$} & \multicolumn{4}{|c|}{$\sigma=50$} \\
\hline & \multicolumn{2}{|c|}{ PSNR } & \multicolumn{2}{|c|}{ SSIM } & \multicolumn{2}{|c|}{ PSNR } & \multicolumn{2}{|c|}{ SSIM } & \multicolumn{2}{|c|}{ PSNR } & \multicolumn{2}{|c|}{ SSIM } \\
\hline & $\begin{array}{l}\text { Mean } \\
\text { Rank }\end{array}$ & $\begin{array}{c}p- \\
\text { Value }\end{array}$ & $\begin{array}{l}\text { Mean } \\
\text { Rank }\end{array}$ & $\begin{array}{c}p- \\
\text { Value }\end{array}$ & $\begin{array}{l}\text { Mean } \\
\text { Rank }\end{array}$ & $\begin{array}{c}p- \\
\text { Value }\end{array}$ & $\begin{array}{l}\text { Mean } \\
\text { Rank }\end{array}$ & $\begin{array}{c}p- \\
\text { Value }\end{array}$ & $\begin{array}{l}\text { Mean } \\
\text { Rank }\end{array}$ & $\begin{array}{c}p- \\
\text { Value }\end{array}$ & $\begin{array}{l}\text { Mean } \\
\text { Rank }\end{array}$ & $\begin{array}{c}p- \\
\text { Value }\end{array}$ \\
\hline BM3D & 1.90 & \multirow{9}{*}{0.000} & 1.47 & \multirow{9}{*}{0.000} & 2.12 & \multirow{9}{*}{0.000} & 1.69 & \multirow{9}{*}{0.000} & 4.96 & \multirow{9}{*}{0.000} & 2.39 & \multirow{9}{*}{0.000} \\
\hline DnCNN & 4.32 & & 5.16 & & 3.56 & & 4.98 & & 3.91 & & 4.26 & \\
\hline $\begin{array}{l}\text { DnCNN- } \\
\text { Enhanced }\end{array}$ & 4.34 & & 5.32 & & 4.03 & & 5.21 & & 3.10 & & 4.00 & \\
\hline BRDNet & 2.13 & & 3.26 & & 2.29 & & 3.25 & & 2.21 & & 2.77 & \\
\hline DHDN & 7.97 & & 7.65 & & 7.81 & & 7.37 & & 7.46 & & 7.09 & \\
\hline CBDNet & 3.75 & & 6.07 & & 4.26 & & 6.38 & & 4.22 & & 5.53 & \\
\hline MuNet & 6.51 & & 3.51 & & 6.13 & & 2.96 & & 5.60 & & 4.66 & \\
\hline EDNet & 5.18 & & 3.87 & & 6.07 & & 4.63 & & 6.06 & & 5.99 & \\
\hline GAN-RW & 8.90 & & 8.67 & & 8.72 & & 8.53 & & 7.49 & & 8.31 & \\
\hline
\end{tabular}

3

4

5

6

7

8

9

10

11

12

13 